\title{
Nano-CT characterization reveals coordinated growth of a rudimentary organ necessary for soldier development in the ant Pheidole hyatti
}

\author{
Sophie Koch ${ }^{1}$, Rui Tahara ${ }^{1}$, Angelly Vasquez-Correa ${ }^{1}$ and Ehab Abouheif ${ }^{1 *}$ \\ ${ }^{1}$ Department of Biology, McGill University, 1205 Avenue Docteur Penfield, Montréal, QC, \\ Canada H3A 1B1 \\ * Corresponding Author: Ehab Abouheif (ehab.abouheif@mcgill.ca)
}

Running title: organ coordination of a rudimentary organ

Keywords: Inter-organ Communication, rudiments, ecoevo-devo, novel ant castes, Pheidole, nano-CT, imaginal disc, wing disc, ant head, ants 
1

\section{Abstract}

The growth of imaginal discs in holometabolous insects is coordinated with larval growth to ensure the symmetrical and proportional development of the adult appendages. In ants, the differential growth of these discs generates distinct castes - the winged male and queen castes and the wingless worker caste. In the hyperdiverse ant genus Pheidole, the worker caste is composed of two morphologically distinct subcastes: small minor workers and larger, bigheaded soldiers. Although these worker subcastes are completely wingless, soldier larvae develop rudimentary forewing discs that are necessary for generating the disproportionate head-to-body scaling of the big-headed soldier subcaste. However, it remains unclear whether rudimentary forewing discs in soldier larvae are coordinated with other imaginal discs, and whether disc growth and coordination patterns vary between the minor worker and soldier subcastes. Here we show, using quantitative nano-CT three-dimensional analyses, that growth of the soldier rudimentary forewing discs is coordinated with the increase in volume of the leg and eye-antennal (head) discs as well as with larval size. We found that the growth rate of the rudimentary forewing discs differs from the leg discs but is similar to the growth of the head (eye-antennal) discs relative to larval size, suggesting that growth of each type of imaginal disc may be differentially regulated. In addition to their larger size, the soldier eye-antennal discs increase in width as they undergo morphogenesis to generate the characteristic shape of the large soldier head, suggesting that the rudimentary forewing discs may regulate their patterning in addition to their growth. Finally, we observe little growth of the leg and eyeantennal discs during the bipotential stage, while in minor worker development these discs grow at similar rates to one another in coordination with larval size to generate the smaller minor worker subcaste. Our results suggest that rudimentary organs with regulatory functions may participate in new patterns of inter-organ coordination and regulation to produce novel phenotypes and complex worker caste systems. We provide characterization of larval development and imaginal disc growth and morphogenesis with the aim of highlighting this as an emerging system for the study of rudimentary organs during development and evolution. 
Introduction

During development, each organ is specified and undergoes growth and differentiation - a process that is intrinsically regulated, yet also coordinated, with that of other organs and with the growth of the whole organism (Andersen et al., 2013; Droujinine \& Perrimon, 2016). This 'inter-organ coordination' is phylogenetically widespread and has been especially well documented in the holometabolous insects, which includes flies, butterflies, beetles, and ants (Andersen et al., 2013; Engel, 2015; Droujinine \& Perrimon, 2016; Busse et al., 2018; Gontijo \& Garelli, 2018; Rosello-Diez et al., 2018; Koch \& Abouheif, 2020). Holometabolous insects have radiated into many ecological niches and their success is thought to be in part due to the evolution of discrete ontogenetic life stages, in which embryos pass through a distinct larval stage before they undergo metamorphosis and transition into their adult form (Truman \& Riddiford, 1999; Yang, 2001). During this larval stage, the presumptive appendages, such as the legs, wings, head structures (including the eyes and antennae), as well as the genital structures,

42 develop from cell populations called imaginal discs (Held, 2002). Imaginal discs are specified

43 during embryogenesis and, during larval development, they proliferate and are patterned by

44 largely conserved gene regulatory networks (Held, 2002). As the larva transitions to the pupal

45 stage and begins the process of metamorphosis, each disc, and its surrounding peripodial

46 membrane, undergo morphogenesis to develop into the specified appendage (Haynie \& Bryant,

47 1986; Fristrom, 1993). Each disc develops autonomously and behaves like a quasi-independent developmental module, yet at the same time, its growth is regulated to coordinate with other discs and with development of the whole larva (Held, 2002; West-Eberhard, 2003; Andersen et al., 2013). Mechanisms that regulate disc growth and coordination maintain developmental robustness and can also be targeted by selection to generate novel morphologies and scaling

52 relationships (Williams \& Carroll, 1993; Emlen \& Nijhout, 2000; Emlen et al., 2012; Vallejo et al., 53 2015; Shingleton \& Frankino, 2018).

Ants are holometabolous insects that have evolved colonies with a morphologically

55 distinct winged queen caste, which primarily performs reproductive tasks, and a wingless

56 worker caste, which primarily performs all other tasks such as foraging, brood-care, and 57 defense (Hölldobler \& Wilson, 2009). Queen and worker castes are polyphenic, which means 
that they are determined during development by environmental cues such as nutrition, temperature, and social interactions (Brian, 1963; Penick \& Liebig, 2012; Lillico-Ouachour \& Abouheif, 2017). In some lineages, the worker caste has evolved inter-individual variation in size and head-to-body size allometry (disproportionate scaling) called 'worker polymorphism' (Wilson, 1953; Fjerdingstad \& Crozier, 2006; Wills et al., 2018). There are multiple types of worker polymorphism, where each type is defined by the distribution of size and head-to-body size scaling of individuals within the colony (Wilson, 1953). Ants in the hyperdiverse genus Pheidole are a classic example of 'dimorphic allometry', where the minor worker and soldier subcastes form two discrete allometric lines (Wilson, 1953; Wilson, 2003). The smaller minor workers generally perform brood-care, nest maintenance, and basic foraging, while larger soldiers contribute more to defense and efficiently perform specific foraging tasks, such as seed-milling (Wilson, 1984; Freener, 1987; Powell \& Clark, 2004; Pfeiffer et al., 2006; Mertl \& Traniello, 2009; Wills et al., 2018). Many studies have examined the morphological, behavioral, and ecological function, as well as the evolvability, of adult morphology in Pheidole worker subcastes, yet much remains to be explored as to how their morphology is generated during development (Wilson, 1984; Pie \& Traniello, 2007; Economo et al., 2015; Holley et al., 2016). growth of imaginal discs (Wilson, 1953). Although adult workers are wingless, worker larvae often develop wing rudiments that vary in their size, shape, and expression of the wing gene regulatory network (Dewitz, 1878; Wheeler \& Nijhout, 1981a; Abouheif \& Wray, 2002; Bowsher et al., 2007; Shbailat \& Abouheif, 2013). In the hyperdiverse genus Pheidole, rudimentary wing disc growth is not activated in minor worker larvae (Figure 1d), while in soldier larvae rudiments of the forewing discs are activated, grow rapidly, and increase together with larval size (Figure 7e) (Wheeler \& Nijhout, 1981a; Abouheif \& Wray, 2002; Sameshima et al., 2004b; Shbailat \&

82 Abouheif, 2013). In comparison, male and queen larvae develop large fore- and hindwing discs

83 that develop into wings used for mating flights (Figure 1b, c) (Abouheif \& Wray, 2002; Keller et

84 al., 2014). While the queen-worker developmental switch occurs during embryogenesis, the 85 soldier-minor worker switch occurs during the late larval stage (Figure 1a) (Passera, 1974; 
87 'bipotential' and can develop into soldiers or minor workers (Wheeler \& Nijhout, 1983;

88 Wheeler, 1986). During this critical window, a high protein diet activates juvenile hormone (JH),

89 which activates soldier development, while if JH fails to surpass its threshold, minor worker

90 development proceeds (Figure 1a) (Passera, 1974; Wheeler \& Nijhout, 1981b, 1983; Rajakumar

91 et al., 2012; Metzl et al., 2018). Soldier rudimentary forewing discs appear following worker

92 subcaste determination in the last larval instar and grow positively and rapidly relative to larval

93 length (Wheeler \& Nijhout, 1981a). However, unlike in queen larvae where leg and wing discs

94 undergo morphogenesis during the larval-to-pupal transition, the growth of the soldier

95 rudimentary forewing discs appears to slow towards the end of the larval stage, becoming

96 uncoupled from the leg discs that begin to undergo morphogenesis (Wheeler \& Nijhout, 1981a;

97 Shbailat et al., 2010). These rudiments then degenerate during the larval-to-pupal transition to

98 form small pupal wing buds, which disappear before the adult stage (Wheeler \& Nijhout, 1981a;

99 Sameshima et al., 2004a; Sameshima et al., 2004b; Shbailat et al., 2010).

100 Even though the rudimentary forewing disc shows remarkable evidence of regulated

101 growth, gene expression, and apoptosis, their appearance and growth have long been thought

102 to occur due to pleiotropic signaling of JH that activates portions of the queen developmental

103 program (Wheeler \& Nijhout, 1981a; Molet et al. 2012, Londe et al.2015, Trible \& Kronauer,

104 2017; Trible \& Kronauer 2020). However, Rajakumar et al. (2018) showed that the appearance

105 of rudimentary forewing discs is not a side-effect, but rather, they function to generate size and

106 disproportionate head-to-body scaling of the soldier subcaste (Figure $2 \mathrm{~g}$, i). Molecular and

107 physical perturbation of the growth of rudimentary forewing discs generates 'intermediates'

108 between wild-type minor workers and soldiers that have altered disproportionate head-to-

109 body scaling. This shows that the growth of the rudimentary forewing discs positively regulates

110 head and body size and is necessary for the development of disproportionate head-to-body

111 scaling in soldiers (Rajakumar et al. 2018). It also suggests that they may participate in a system

112 of inter-organ regulation. However, Rajakumar et al. (2018) results differ from previous

113 observations where perturbations to disc growth result in (1) no detectable adult phenotype

114 due to homeostatic regulation between the damaged disc and other developing discs; or in (2)

115 an increase in the mass of the other adult organs due to differential resource allocation and 
compensatory growth (Klingenberg \& Nijhout, 1998; Nijhout \& Emlen, 1998; Parker \&

117 Shingleton, 2011; Andersen et al., 2013). Therefore, the regulation between the rudimentary

118 forewing and other imaginal discs in the genus Pheidole may be a unique or novel system of

119 inter-organ coordination through which the rudimentary forewing discs generate

120 disproportionate head-to-body allometry (Rajakumar et al. 2018).

121 The main goal of this study is to examine and characterize how the rudimentary

122 forewing discs in soldiers participate in a potentially novel system of inter-organ coordination

123 and how disc growth and coordination vary across worker subcastes. Therefore, in Pheidole

124 hyatti, we characterized how the growth of rudimentary forewing discs in soldiers correlates

125 with the growth of the leg and head (eye-antennal) discs within a larva to generate

126 disproportionate head-to-body scaling and how the leg and eye-antennal discs grow and are

127 coordinated with larval size during the bipotential and minor worker stages. The eye-antennal

128 discs give rise to much of the adult head, which differs significantly in size and shape between

129 worker subcastes (Schoeller, 1964; Garcia-Bellido \& Merriam, 1969; Milner \& Haynie, 1979;

130 Haynie \& Bryant, 1986; Wilson, 2003). To investigate these questions, we used X-ray nano-

131 computed tomography (nano-CT) to reconstruct the leg, eye-antennal, and rudimentary

132 forewing discs throughout late larval development. Unlike dissection preparations, nano-CT

133 permits us to (1) characterize multiple organs in whole undissected larva; (2) characterize

134 organs, such as the eye-antennal discs, in three dimensions (3D) because their shape and

135 location render them difficult to quantify in two dimensions (2D); and (3) characterize organs

136 immediately before the larval-to-pupal transition when discs begin to undergo morphogenesis

137 and detach from the larval cuticle (Hall et al., 2017; Watanabe et al., 2017; Schoborg et al.,

138 2018). First, we determined the timing of subcaste determination during larval development

139 and the number of larval instars. We then used nano-CT to examine the growth of the imaginal

140 discs prior to and following the soldier-minor worker developmental switch. The

141 characterization of imaginal disc development by nano-CT will shed light on how a rudimentary

142 organ - the rudimentary forewing discs in Pheidole hyatti is coordinated with the growth of the

143 leg and eye-antennal imaginal discs during larval development to generate disproportionate 
144 head-to-body scaling and how differential disc growth generates complex worker caste

145 systems.

146 Materials and Methods

147 Ant collection and care

148 Queen-right colonies of Pheidole hyatti were collected near Globe, Arizona, USA. Colonies were 149 housed at $27^{\circ} \mathrm{C}$ and $60 \%$ humidity on a 12 hour light:dark cycle in the Conviron environmental 150 chambers at the McGill University Phytotron. Nests were constructed from fluon-coated plastic 151 bins with several water-filled glass tubes and a single sugar-water filled glass tube plugged with 152 cotton. Queen-right colonies were fed Bhatkar-Whitcomb yellow diet (Bhatkar \& Whitcomb, 153 1970) and frozen mealworms $3 X$ a week and were supplemented with frozen Drosophila hydei 154 1-2X a week.

156 Larval development and scanning electron microscopy (SEM)

157 To quantify the number of larval instars, larvae were randomly collected from several 158 established colonies and imaged on their dorsum using a Zeiss Discovery V12 stereomicroscope. 159 Larval head capsule width (from the lateral edges of the occipital border) and larval length were 160 measured. These were chosen as morphological landmarks as they have previously been shown 161 to identify: (1) changes in larval head capsule width and larval length associated with larval 162 molts, and (2) changes in larval length associated with worker polymorphism (Wheeler \& 163 Nijhout, 1981a; Solis et al., 2010; Alvarado et al., 2015). Larvae designated 'minor worker'

164 (Figure $2 \mathrm{a}, \mathrm{f}, \mathrm{g}$ ) or 'soldier' (Figure 2a, h, i) are terminal larvae immediately before the larval-to165 pupal transition are characterized by a dark brown-black gut and changes in fat distribution 166 (Wheeler \& Wheeler, 1976). Before this stage, larvae in the first to third instar and the early 167 fourth instar have a light brown gut (Passera, 1974). During the fourth instar, the gut becomes 168 darker brown until it blackens immediately before the prepupal stage. We use larval length as a 169 proxy for the developmental stage and maturation of the larva within this instar (Passera, 170 1974). All measurements were performed using Zeiss AxioVision Software v4.9.1. Scanning 171 electron microscopy was done on a Hitachi TM3030 Plus TableTop Scanning Electron

172 Microscope. Larval and pupal scans were performed on live specimens. 
174 Nano-Computed Tomography (nano-CT) scanning and reconstruction

175 Larval samples were collected from a single established colony, measured for larval length, and 176 samples were fixed for imaginal disc characterization. As nano-CT analyses are costly and time-

177 intensive, we were limited in our assays to 3 brown gut bipotential larvae (Figure 1a, grey line),

1782 brown gut minor worker-destined larvae (Figure 1a, black line), 7 brown gut soldier-destined

179 larvae (Figure 1a, green line), and one black gut terminal larvae of each subcaste just before the 180 larval-to-pupal transition (Table 1). Larvae were assigned to stages and subcastes based on 181 larval length, morphology, and gut colour (Figure 2a) (Passera, 1974; Wheeler \& Wheeler, 182 1976). Minor worker and soldier prepupae were fixed in the same way as larvae. All samples 183 were rehydrated to PBS-Tw(0.1\%), dehydrated to $70 \% \mathrm{EtOH}$, and then stained for 4-21 days in 184 1\% phosphotungstic acid (PTA; Abcam, ab146206-25G) in 70\% EtOH depending on the size of 185 the sample (Metscher, 2009a, b). A small hole was punctured through the cuticle of a 186 representative queen and male samples towards the posterior of the larvae to prevent 187 shrinking during staining. Samples were embedded either in $0.5 \%$ agarose in a microcentrifuge 188 tube or in $70 \% \mathrm{EtOH}$ in a $200 \mu$ l pipette tip. Images were acquired on a Zeiss Xradia 520 Versa 189 (Carl Zeiss Canada Ltd., ON, Canada) with a resolution ranging from $0.7 \mu \mathrm{m}$ to $3.6 \mu \mathrm{m}$ using $4 \mathrm{X}$ or $19020 X$ objective lens and single FOV mode. The source and secondary filters were chosen based 191 on the sample. 3201 projections were taken for each specimen over 360 degree-scan. Scans 192 were focused on the anterior half of the larvae to visualize the anterior imaginal discs at high 193 resolution. Detailed information of sample preparation and image acquisition parameters is in

194 Table 1. All three-dimensional reconstructions were done on Imaris x64 v9.2.1 (Bitplane AG, 195 Switzerland). Individual imaginal discs, including their peripodial membrane, and larval brain 196 were segmented and surfaces were filtered manually using 2-6 $\mu \mathrm{m}$ resolution to ensure that the 197 disc and peripodial membrane were included, while other larval tissues were excluded in the 198 surface. Volumes were calculated using the Imaris statistics tool. In regions where adjacent 199 discs began to fuse, the surface cutting tool was used to separate them. Prepupal samples were 200 segmented as a single 3D surface then false coloured to capture the entirety of their 201 morphology. 
Soldier rudimentary forewing disc area measurements

Soldier larvae were collected randomly from several established lab colonies based on larval size and gut colour characteristics. Larvae were imaged for larval development characterization

207 and measured for larval length, then fixed for immunohistochemistry and counter-stained with

208 DAPI before flat mounting in 50\% glycerol. Disc area measurements were performed to exclude 209 the peripodial membrane on a Zeiss Axiolmager Z1 microscope using Zeiss AxioVision software

210 v4.9.1. Measurements were eliminated due to disc tearing or poor boundary visibility.

\section{Statistics}

213 All statistical analyses were performed in Prism v8 and we considered $\mathrm{P}<0.05$ statistically

214 significant. For multiple comparisons, our level of significance was corrected using a Bonferroni

215 correction. All measures of area and volume were log-transformed before analysis

216 (untransformed volumetric estimations can be found in Figure 8). ANCOVA analysis was used to 217 compare the slopes of linear regressions for average imaginal disc area or volume relative to 218 larval length or leg disc volume. If no difference in slope was detected, ANCOVA was used to 219 compare the $y$-intercepts. We note that our small and unequal sample size between subcastes

220 puts us at risk of Type 2 errors, therefore any non-significant results were interpreted with 221 caution.

\section{Results}

224 Larval development and worker subcaste differentiation

225 To characterize and identify the number of larval instars and size variation within them, we 226 measured larvae for larval head capsule and larval length (Solis et al., 2010). In P. hyatti, these 227 measurements identified four larval instars (Figure 2a). The first instar ranges from 376-438 $\mu \mathrm{m}$ 228 in larval length, the second instar ranges from $448-586 \mu \mathrm{m}$, the third instar ranges from 527$229932 \mu \mathrm{m}$, and the fourth (final) instar, which shows significant variation in larval length including 230 bipotential, minor worker, and soldier larvae, ranges from 830-3096 $\mu$ m (Figure 2a-f, h). (Figure 
231 2a). The younger larvae in this instar are 'bipotential' (Figure 1a, gray line, and 2e) and have the

232 capacity to develop either into soldiers (Figure 1f) or minor workers (Figure 1h). Following the

$233 \mathrm{JH}$-mediated developmental switch, the minor worker and soldier developmental programs

234 proceed and differentiate. As larvae approach the end of larval development, the light brown

235 gut continues to darken until the terminal stage, at which time, it becomes dark black, and

236 larvae show changes in fat distribution (Passera, 1974; Wheeler \& Wheeler, 1976). Minor

237 worker larvae become terminal between 1804-1998 $\mu \mathrm{m}$ (Figure 2h), whereas soldier larvae

238 grow larger and become terminal between 2666-3096 $\mu \mathrm{m}$ (Figure 2f). Before the terminal stage,

239 larvae are 'minor worker-destined' (Figure 1a, black line) or 'soldier-destined' (Figure 1a, green

240 line) and have a light brown gut. Differentiation within the fourth instar produces two discrete

241 clusters of terminal stage larvae (Figure 2a). These terminal clusters together generate the

242 discrete dimorphic head-to-body allometry that is characteristic of typical Pheidole worker

243 caste systems (Figure $2 \mathrm{~g}, \mathrm{i}$ ).

245 Quantitative nano-CT analyses of imaginal disc growth and coordination

246 We describe the growth and morphogenesis of these imaginal discs and compare the slope and

$247 \quad y$-intercepts of their linear regressions within subcastes to investigate their growth relative to

248 larval length and relative to leg disc volume.

251 In the bipotential stage (830-1600 $\mu \mathrm{m}$ in larval length), larvae have the capacity to develop into

252 soldiers or minor workers and can be experimentally induced to a soldier fate by exogenous

253 application of a JH-analog (Wheeler \& Nijhout, 1983; Rajakumar et al., 2012). During this stage,

254 the leg discs are spherical (Figure 3) and their volume does not increase significantly relative to

255 larval length $(F=1.660, d f=1, P=0.4202)$ (Figure $7 a, d)$. The three pairs of leg discs are positioned

256 ventrally, inferior to the larval head capsule and lateral to the ventral nerve cord. The eye-

257 antennal discs also begin as spherical discs and are positioned anterosuperior to the larval brain

258 within the larval head capsule (Figure 3). Throughout bipotential development, the eye-

259 antennal discs become slightly more oblong and extend anteriorly (Figure 3). During this stage, 
the volume of the eye-antennal discs does not correlate with larval length $(F=4.436, d f=1$, eye-antennal discs do not differ significantly in slope or y-intercept compared to the leg discs (slope: $F=0.6733, \mathrm{df}=2, \mathrm{P}=0.4982$; $\mathrm{y}$-intercept: $\mathrm{F}=7.104$, $\mathrm{df}=3, \mathrm{P}=0.0760$ ) (Figure $7 \mathrm{~d}$ ) suggesting

264 that the slight growth of the leg and eye-antennal discs occurs at a similar rate during the

265 bipotential stage but does not correlate with larval length. We were unable to detect

266 rudimentary forewing or hindwing discs in bipotential samples (Figure 3).

Coordination of imaginal disc development in minor worker larvae

269 During minor worker development (1600-1998 $\mu \mathrm{m}$ in larval length), the leg discs increase positively in volume relative to larval length (Figure 7a, d). In terminal larvae, the legs begin to

271 undergo morphogenesis, increase significantly in volume, and their peripodial membranes

272 begin to evert and merge with those of the adjacent discs (Figure 4c, c'). Similar to the leg discs,

273 the eye-antennal discs increase positively in volume relative to larval length (Figure 7b, d) and

274 increase positively relative to leg disc volume (Figure 7e). The eye-antennal discs become more

275 oblong, extending anteriorly into the larval head capsule as minor worker development

276 progresses (Figure 4a, b, a', b'). The increase in volume of the eye-antennal discs continues

277 until, at the end of minor worker larval development, they undergo morphogenesis: the

278 presumptive antenna evert, and the peripodial membranes fuse along the midline, expanding

279 laterally to envelop the larval brain (Figure 4c, c'). In minor worker larvae, eye-antennal discs

280 have a significantly larger y-intercept compared to leg disc volume but do not differ in slope

281 (slope: $F=16.15, d f=2, P=0.0567$; $y$-intercept: $F=22.24, d f=3, P=0.0181$ ) (Figure 7d). We observed

282 very small forewing rudiments in one minor worker-destined sample (Figures $4 b, b^{\prime}$ and $7 c$ ) that

283 were no longer detectable by the terminal stage (Figure 4c, c').

We were able to visualize discs near the larval mouthparts in the terminal minor worker

285 sample that appear to be derived from the labial and/or clypeo-labral discs that contribute to

286 the mandibles and other mouthparts (Figure 4c, c') (Kumar et al., 1979; Held, 2002). At the

287 stage just prior to this $\left(b, b^{\prime}\right)$, the distinct mass (labeled white) under the main larval brain (also

288 labeled white) may correspond to the labial and/or clypeo-labral disc before its differentiation 
into clear mouthparts. However, the resolution of our scan was not sufficient to confirm whether this distinct mass represents undifferentiated mouthparts. At the terminal stage, these discs then merge with the eye-antennal discs and appear to vary in shape between subcastes

292 (Figure 8a-b'). By the prepupal stage, the mandibles and other mouthparts are more clearly

293 visible (Figure 8c-d'). Based on the prepupal scans that we performed, we estimated the

294 boundary between the presumptive mandibles and other mouthparts from the eye-antennal

295 discs in our terminal samples and then removed the former from our estimation of the eyeantennal discs as they are likely derived from other discs (Figure 8).

Coordination in imaginal disc development in soldier larvae

299 In addition to a larger terminal size compared to minor workers, soldier-destined larvae are 300 characterized by the growth of the rudimentary forewing discs (Figure 6). The soldier rudimentary forewing discs differ considerably from the queen and male forewing discs in size 302 and shape, and hindwing rudiments are undetectable (Figure 1b, c, e). To estimate how the rudimentary forewing discs grow relative to the leg discs in soldier larvae, we first measured 304 the area of the discs relative to larval length using standard dissection techniques. The three pairs of leg discs increase in area positively relative to larval length and there is no significant difference in slope or $y$-intercept between the average disc area across the three thoracic segments (slope: $F=0.2961, d f=2,113, P=0.7443$; $y$-intercept: $F=0.3184, d f=2,113, P=0.7280$ )

(Figure 5a). Using nano-CT 3D analyses, we observed that in soldier-destined larvae with approximately the same larval length as the terminal minor worker larvae the leg discs remain

311 increase positively in volume relative to larval length (Figure 7a, d), acquiring visible segments

312 along their proximo-distal axes (Figure 6a-g, $a^{\prime}-g^{\prime}$ ). Similar to minor workers, as the soldier

313 larvae approach the larval-pupal transition, the leg discs undergo morphogenesis, increase

314 significantly in volume, and detach from the larval cuticle (Figure 6h, $h^{\prime}$ ). By the end of larval

315 development, the average leg disc volume in the terminal soldier sample is approximately 2.5

316 times larger than the average leg disc volume of the minor worker sample (Figure 7a). 
During soldier development, the eye-antennal discs continue to grow in volume and

318 length, extending distally into the larval head capsule (Figure 6a-g, a'-g'). The peripodial

319 membranes that surround the eye-antennal discs begin to fuse along their medial edges until,

320 at the late soldier-destined stage, the membranes further expand laterally around the larval

321 brain (Figure 6g, $g^{\prime}$ ). Throughout soldier development, eye-antennal disc volume correlates

322 positively with larval length and leg disc volume (Figure 7b, d, e). Relative to larval length, the

323 soldier eye-antennal discs are larger and differ significantly in y-intercept compared to the leg

$324 \operatorname{discs}(F=8.460, d f=13, P=0.0122)$ but do not differ in slope $(F=0.03928, d f=12, P=0.8462)$ (Figure

$3257 \mathrm{~d})$. In the terminal soldier larvae, the eye-antennal discs also undergo morphogenesis and are

326 approximately 1.43 times larger in volume than those in minor workers (Figures $6 \mathrm{~h}, \mathrm{~h}^{\prime}$ and $7 \mathrm{~b}$ ).

327 At the terminal stage, the peripodial membrane expands to surround the larval brain and forms

328 the presumptive adult head. In addition to being larger in volume, the width of the peripodial

329 membrane is larger in soldiers than minor workers and appears to further enlarge during the

330 prepupal stage (Figure 8b, $b^{\prime}, d, d^{\prime}$ ). Just as in our terminal minor worker sample, we removed

331 the presumptive mandibles and mouthparts from the estimation of the eye-antennal discs in

332 the terminal soldier sample.

The rudimentary forewing discs begin as a small cluster of cells in the early soldier-

334 destined stage and increase in volume rapidly throughout the soldier-destined period,

335 obtaining a more triangular shape in lateral view in late larval development (Figures 6 and 7c).

336 However, there is some plasticity in the overall ontogenetic trajectory between developing

337 soldier larvae (Figure 6e-g). The soldier rudimentary forewing discs generally increase in both

338 area and volume relative to larval length and relative to leg disc volume (Figures 5b and 7).

339 Relative to larval length, the area of soldier rudimentary forewing discs increases at a

340 significantly different rate compared to the average leg disc area $(F=11.51, d f=82, P=0.011)$

341 (Figure 5b) and the volume of the soldier rudimentary forewing discs also increases at a

342 significantly different rate compared to the volume of the soldier leg discs $(F=8.806, d f=12$,

$343 \mathrm{P}=0.0118)$. The increase of soldier rudimentary forewing disc volume is not significantly

344 different from the soldier eye-antennal disc volume (slope: $F=5.879, d f=12, P=0.0320, y$ -

345 intercept: $F=7.053, \mathrm{df}=12, \mathrm{P}=0.0210$ ) (Figure 7d). However, when plotted relative to leg disc 
volume, soldier rudimentary forewing disc volume increases at a significantly different rate compared to the soldier eye-antennal $\operatorname{discs}(\mathrm{F}=17.31, \mathrm{df}=12, \mathrm{P}=0.0013)$ (Figure 7e). In terminal

348 larvae, the rudimentary forewing discs detach from the larval cuticle and remain attached to 349 the larval fat body through their peripodial membrane (Figure 6h, h'), however, unlike the leg 350 and eye-antennal discs, they do not undergo morphogenesis.

\section{Discussion}

353 The growth of the soldier rudimentary forewing discs positively regulates the growth of head 354 and body size to generate disproportionate head-to-body scaling and the soldier phenotype in 355 P. hyatti (Rajakumar et al. 2018). These results suggest that these rudiments may regulate the 356 differential growth of the other discs through a potentially novel system of inter-organ 357 coordination. Here we describe the growth and development of three imaginal discs and show 358 that the activation of the rudimentary forewing discs is coordinated with an increase in the 359 volume of the leg and eye-antennal discs and with an increase of larval size. Furthermore, as 360 the soldier eye-antennal discs undergo morphogenesis, they increase in width to specify the 361 larger soldier head and generate its characteristic shape. In contrast, little imaginal disc growth occurs during the bipotential stage, whereas the leg and eye-antennal discs increase proportionally in minor worker development. Collectively, this shows stage and subcastespecific differences in disc growth and morphogenesis. This study provides a foundation for understanding how these rudiments grow and participate in inter-organ coordination to generate disproportionate head-to-body scaling. More generally, we highlight the importance of characterizing development across stages and subcastes to investigate the evolution of complex worker caste morphology in ants.

The developmental differentiation that generates worker polymorphism occurs during

370 the last larval stage (Wheeler \& Nijhout, 1981a, 1983; Sameshima et al., 2004b; Alvarado et al., 371 2015). In P. hyatti and other species of Pheidole, this differentiation produces discrete clusters 372 of terminal larvae that generate the dimorphic minor workers and soldiers that both develop 373 from the early bipotential stage. This contrasts with the carpenter ant, Camponotus floridanus, 374 which has a continuous worker caste system of minors, media, and major workers, and whose 
375 fourth instar is represented by early bipotential larvae and a continuous spread of terminal

376 larval sizes (Alvarado et al., 2015). This shows that modifications to larval growth and terminal

377 size can generate different types of worker polymorphism in ants (Wilson, 1954). The

378 developmental mechanisms that underlie the differences and distributions of larval size within

379 a worker caste system involve $\mathrm{JH}$, nutrition-based signals, such as the insulin/insulin-like

380 pathways, social pheromone-based regulation, and/or epigenetic modifications, yet much

381 remains to be explored as to how these signaling pathways interact during larval development

382 and vary between complex caste systems (Wheeler \& Nijhout, 1984; Wheeler, 1986; Rajakumar

383 et al., 2012; Alvarado et al., 2015; Corona et al., 2016; Lillico-Ouachour \& Abouheif, 2017).

384 While larval length correlates positively with adult body size, it does not entirely explain

385 changes in disproportionate head-to-body scaling (Wilson, 1953; Alvarado et al., 2015).

386 Therefore, we focused our characterization on the differential growth and morphology of the

387 larval imaginal discs, the precursors of the adult structures in holometabolous insects

388 Our results show that, following soldier subcaste determination, the growth of the

389 rudimentary forewing discs is activated, while the leg and eye-antennal discs continue to grow

390 and reach a final larger size, which is correlated with an increase in larval size. This shows that

391 the growth of the soldier rudimentary forewing discs is coordinated with the other imaginal

392 discs and with larval growth. Within a larva, smaller rudimentary forewing discs co-occur with

393 smaller leg and eye-antennal discs, suggesting that coordination of growth between discs may

394 be more constrained compared to their coordination with overall larval size. Each disc's growth

395 trajectory may be determined by organ-specific sensitivity to systemic endocrine signals, such

396 as JH or insulin, or due to endogenous properties of the disc (Emlen et al., 2012). The

397 rudimentary forewing discs grow more rapidly compared to the leg discs, which is consistent

398 with ours and previous results using area to estimate disc growth (Wheeler \& Nijhout, 1981a).

399 However, we observe that the growth of the rudimentary forewing discs does not differ

400 significantly from the growth of the eye-antennal discs relative to larval length. This pattern

401 may be a result of the intimate relationship between the rudimentary forewing discs and head

402 growth. This is consistent with Rajakumar et al.'s (2018) finding that perturbations to

403 rudimentary forewing disc growth have a more significant effect on pupal head size than body 
size. However, we cannot rule out that this non-significant relationship between the growth of the rudimentary forewing discs and eye-antennal discs is a result of our low sample size, and thereby, low statistical power. Future studies can investigate this further as well as examine

407 how perturbations to the growth of the solider rudimentary forewing discs affect the growth of 408 the leg and eye-antennal discs.

While the relationship between the leg and rudimentary forewing discs has been

410 previously examined in $P$. bicarinata, the growth of the eye-antennal discs in ant worker

411 subcastes has not been well explored despite the notable phenotypic differences in adult head

412 morphology. We observed that the eye-antennal discs and leg discs increase in volume at

413 similar rates relative to one another during soldier development. However, as the eye-antennal

414 discs undergo morphogenesis, they appear to increase in width as they envelop the larval brain

415 and generate a wide and morphological distinct head cuticle by the prepupal stage. Previous

416 work suggests that modifications to the patterning and the size of the eye-antennal discs may

417 be the source of generating variation in the relative scaling of organs on the head (Dominguez

418 \& Casares, 2005). This suggests that the activation of the growth of the soldier rudimentary

419 forewing discs and the soldier developmental program may regulate the increase in eye-

420 antennal disc volume as well as regulate their patterning to specify a larger region of head

421 cuticle and modifications to the antennae and eyes. In contrast, we observed no comparable

422 differences in the leg discs between subcastes other than their size. Therefore, as the soldier

423 rudimentary forewing discs are necessary to produce the solider phenotype, our results suggest

424 that the activation of the soldier developmental program and the rapid growth of the

425 rudimentary forewing discs causes the leg and eye-antennal discs to grow larger throughout

426 soldier development. Furthermore, they may also regulate the patterning of the eye-antennal

427 discs to generate the soldier head shape as the discs undergo morphogenesis.

428 We observed that during the bipotential stage before worker subcaste differentiation,

429 little disc growth occurs and, while the eye-antennal discs are larger than the leg discs, neither

430 is correlated with larval size. Within our sampled larvae in the minor worker developmental

431 stage, the leg and eye-antennal discs also grow at similar rates but both grow positively relative

432 to larval size. We were unable to detect wing rudiments in our bipotential samples and the 
433 majority of our minor worker samples. However, we observed very small wing buds in one 434 sample that we classified as minor worker-destined based on larval size. In addition to the 435 presence of rudimentary forewing discs in soldiers of $P$. megacephala, Sameshima et al. (2004) 436 observed very small populations of forewing cells in minor worker larvae. Unlike in soldiers, 437 these forewing cells in P. megacephala are never activated, do not undergo significant growth, 438 and appear to not be coordinated with other discs or with larval growth. These authors also 439 observed forewing cells in a high percentage of larvae smaller than the smallest terminal minor 440 workers, which shows that these cells exist but that their growth is not activated in bipotential 441 or minor worker-destined larvae (Sameshima et al., 2004b). This suggests that either that very 442 small clusters of wing cells develop in the bipotential stage and we were unable to detect them 443 or that Sameshima et al. (2004) also observed these discs in minor worker-destined larvae. 444 Alternatively, this sample may represent a very young soldier-destined larva just after soldier 445 determination. During early destined stages, soldier and minor worker larvae are 446 indistinguishable based on larval characteristics but become distinguishable when the minor 447 worker larval gut begins to darken, while the soldier larval gut remains light and soldier larvae 448 continue to develop. We were unable to resolve these possibilities due to our limited sample 449 size and we acknowledge that our low sample size of bipotential and minor worker-destined 450 larvae make it difficult to conclusively establish the patterns of disc growth and compare 451 statistically between subcastes. However, these results preliminarily show that following 452 worker caste determination, differential growth of imaginal discs and larval development 453 generates discrete worker subcaste morphologies. Future studies can further examine these 454 hypotheses by examining more samples across these stages of development. Inter-organ coordination appears to be widespread in insects (Koch \&Abouheif,2020).

456 Two well-described models have been proposed in the literature: homeostasis and resource 457 competition. According to the inter-organ homeostasis model, the coordination of disc growth 458 functions to ensure developmental symmetry. When a disc is damaged, this regulation 459 coordinates the growth of the other discs with the damaged disc and larval development to 460 allow regeneration, producing no adult phenotype or shows no effects on larval development if 461 the disc is eliminated (Madhavan \& Schneiderman, 1969; Parker \& Shingleton, 2011; Hariharan, 
462 2012; Gontijo \& Garelli, 2018). In contrast, according to the inter-organ resource competition

463 model, damage to one or multiple discs results in an asymmetric increase of the mass of the

464 other appendages suggesting that each disc competes locally for resources (Klingenberg \&

465 Nijhout, 1998; Nijhout \& Emlen, 1998). In P. hyatti, however, perturbations to the growth of the

466 soldier rudimentary forewing discs result in a disproportionate reduction of the growth of the

467 head and body size (Rajakumar et al. 2018). As this differs from previous observations of

468 coordination, this suggests that the soldier rudimentary forewing discs have acquired a novel

469 function. Our characterization of the growth relationships of the leg, eye-antennal, and

470 rudimentary forewing discs supports the hypothesis that they participate in a potentially novel

471 system of inter-organ coordination. While it remains to be explored how these rudiments signal

472 and regulate the growth of the head and body size, previous work on queen and worker castes

473 has shown that imaginal discs growth can be modified to generate caste variation. In the ant

474 Myrmecina nipponica, growth of the compound eye, forewing, and hindwing discs diverge in

475 growth and final size between queens, workers, and a novel reproductive caste called ergatoid

476 queens showing that disc growth is modular (Miyazaki et al., 2010). This demonstrates that in

477 ant evolution, modifications to the growth and coordination relationships between discs and

478 larval ontogeny generate the developmental differentiation of castes. These ancestral

479 mechanisms of modularity and plasticity could have facilitated the acquisition of a novel

480 regulatory role by the soldier rudimentary forewing discs in P. hyatti. Furthermore, the growth

481 of rudimentary wing discs varies across other species with complex worker caste systems, in

482 which minor worker larvae develop small wing pads and soldiers develop larger rudimentary

483 wing discs and show inter-specific differences across species that lack soldiers yet develop

484 larval wing rudiments (Abouheif \& Wray, 2002; Bowsher et al., 2007; Shbailat \& Abouheif,

485 2013; Rajakumar et al., 2018). Therefore, the diversity of morphology and elaboration of

486 rudimentary wing discs across ants presents a useful system to investigate the origin, growth,

487 and repurposing of rudimentary organs during development and evolution.

488 Collectively, this study shows how alterations to the growth trajectories of imaginal

489 discs, including a rudimentary organ, by environmental cues can generate discrete and highly

490 adaptive morphologies in ants. We show that the growth of the soldier rudimentary forewing 
491 discs is coordinated with the other discs and may have differential regulatory functions over the

492 growth of the leg and eye-antennal discs and may regulate eye-antennal disc morphogenesis to

493 generate disproportionate head-to-body scaling in the soldier subcaste in P. hyatti. By

494 characterizing and describing the developmental processes that underlie these morphologies,

495 we can begin to elucidate the processes through which worker subcastes differentiate and how

496 disproportionate head-to-body allometry is generated during development. This highlights the

497 emerging properties of this system for the study of rudimentary wing discs and their functional

498 role in the development and evolution of worker caste systems across ants. More generally,

499 future studies should examine the developmental underpinnings of the elaboration and

500 function of rudimentary organs may reveal that they play a role in the regulation of novel

501 phenotypes during development and evolution. Furthermore, future studies should also

502 capitalize on nano-CT as a powerful technique to reveal the larval origin of many key adult

503 features in ants, such as eyes, antennae, head capsule, mandibles, thorax, legs, and genitals,

504 that are often elaborated and differentially expressed between queen and worker castes.

\section{Acknowledgments}

507 We dedicate this manuscript to our collaborator Dr. Tomonari Kaji, a phenomenal researcher

508 who passed away in May 2019 prior to the completion of this work. We also thank Bob Johnson

509 for help collecting ants, Dr. Roberto Keller, and the Abouheif Lab for comments on this

510 manuscript. This research was performed using the infrastructure of the Integrated

511 Quantitative Biology Initiative (IQBI), Canadian Foundation of Innovation project 33122. We

512 thank the McGill Cell Imaging and Analysis Network (CIAN) and the IQBI for equipment support

513 and the Advanced Biolmaging Facility (ABIF) for training. This work was funded by a National

514 Sciences and Engineering Research Council of Canada (NSERC) Discovery Grant to E.A. and an

515 NSERC Canada Graduate Scholarship (Master's) and Fonds de Recherches de Quebec - Nature

516 et Technologies (FRQNT) Scholarship to S.K. 


\section{Table 1. Larval and prepupal sample preparation and image acquisition parameters for nano-}

519 CT scans. $\mathrm{MW}=$ minor worker, $\mathrm{SD}=$ soldier

520

521

\begin{tabular}{|c|c|c|c|c|c|c|}
\hline Stage / subcaste & $\begin{array}{l}\text { Larval length } \\
\qquad(\mu \mathrm{m})\end{array}$ & $\begin{array}{l}\text { Staining } \\
\text { (Days) }\end{array}$ & kV & $\mu \mathrm{A}$ & Voxel size & Exposure (s) \\
\hline bipotential & 1033.43 & 5 & 60.34 & 82.13 & 0.75 & 3 \\
\hline bipotential & 1249.69 & 5 & 60.34 & 82.09 & 0.67 & 6.5 \\
\hline bipotential & 1527.61 & 5 & 60.35 & 82.14 & 0.80 & 2.8 \\
\hline MW-destined & 1634.56 & 5 & 60.34 & 82.12 & 1.102 & 2 \\
\hline MW-destined & 1728.86 & 5 & 60.35 & 82.09 & 1.01 & 2.3 \\
\hline MW terminal & 1915.25 & 4 & 60.34 & 82.08 & 1.00 & 2.5 \\
\hline SD-destined & 1923.49 & 7 & 60.34 & 82.07 & 1.21 & 1.5 \\
\hline SD-destined & 2141.26 & 4 & 60.34 & 82.12 & 1.21 & 2.6 \\
\hline SD-destined & 2301.25 & 7 & 60.35 & 82.12 & 1.30 & 1.3 \\
\hline SD-destined & 2419.53 & 11 & 60.35 & 82.03 & 1.41 & 1.3 \\
\hline SD-destined & 2583.54 & 9 & 60.34 & 82.19 & 1.51 & 1.8 \\
\hline SD-destined & 2614.47 & 9 & 60.34 & 82.08 & 1.40 & 1.7 \\
\hline SD-destined & 2783.18 & 10 & 60.34 & 82.18 & 1.50 & 1.2 \\
\hline SD terminal & 2869.90 & 4 & 60.35 & 82.21 & 1.61 & 1.5 \\
\hline male & - & 21 & 60.35 & 82.13 & 3.61 & 0.4 \\
\hline queen & - & 13 & 60.35 & 82.05 & 4.91 & 0.8 \\
\hline SD prepupae & - & 20 & 60.35 & 82.25 & 1.61 & 0.8 \\
\hline MW prepupae & - & 20 & 60.35 & 82.19 & 1.11 & 1.5 \\
\hline
\end{tabular}

522

523 
a

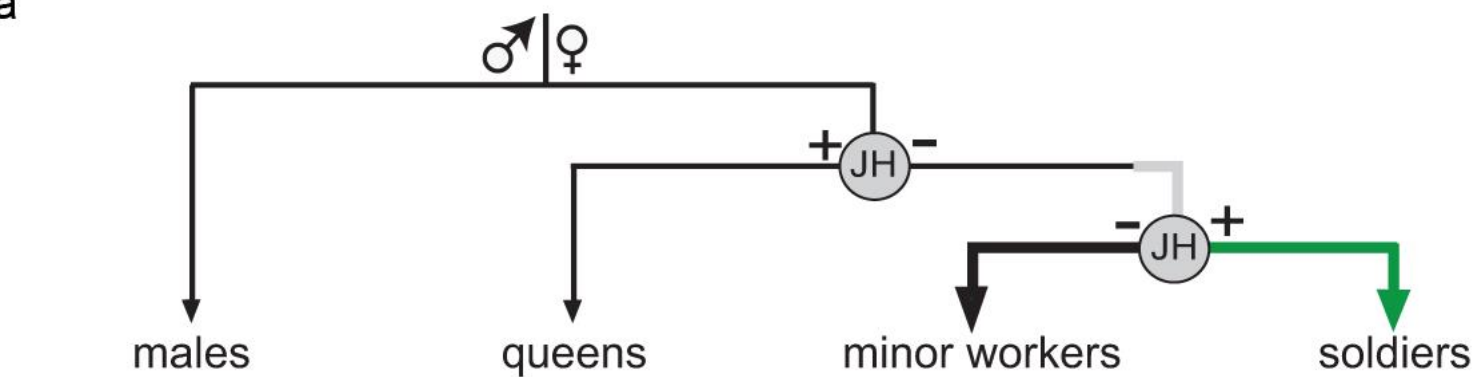

b

\section{C \\ c}

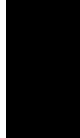

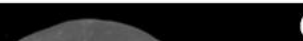

queens

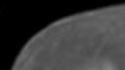

$\sqrt{3}$

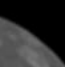

\begin{abstract}
d
\end{abstract}

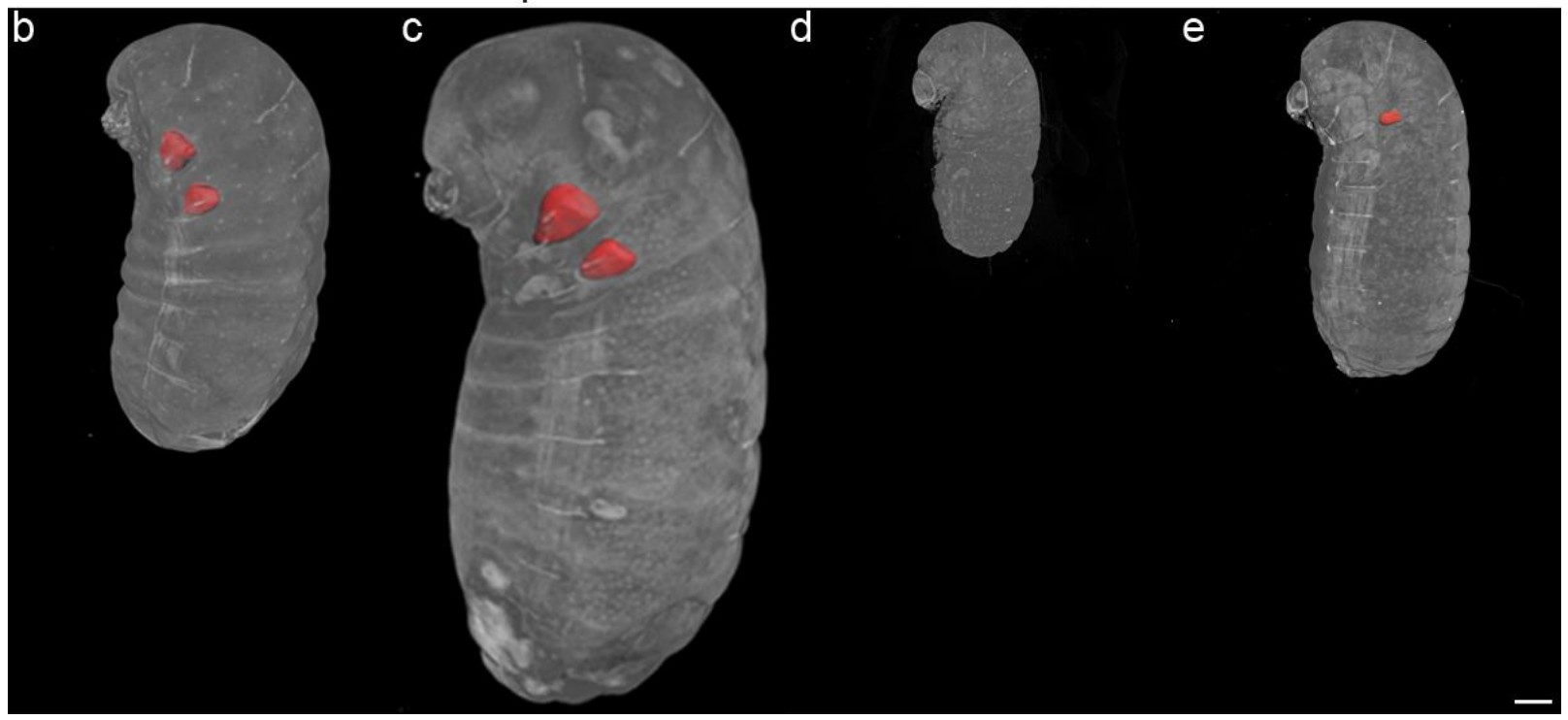

e

Figure 1. Development and caste determination in Pheidole. a) Castes are determined by three developmental switches: 1) the male-female switch is determined by haplo-diploidy during oogenesis, 2) the queen-worker switch during embryogenesis is determined by $\mathrm{JH}$, and 3) the soldier-minor worker switch during larval development is determined by nutrition and JH. Thick grey line represents the bipotential stage, thick black line represents minor worker development, and thick green line represents soldier development. Representative 3D rendered nano-CT images of larvae with fore- and hindwing or rudimentary forewing discs highlighted (red) of b) male, c) queen, and e) soldier. Scale bar indicates $300 \mu \mathrm{m}$. 
a

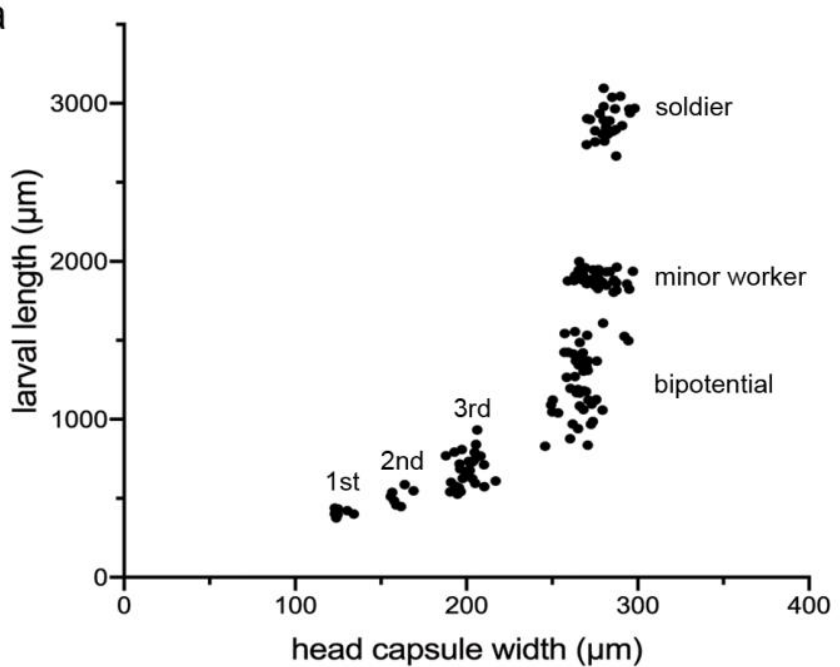

b

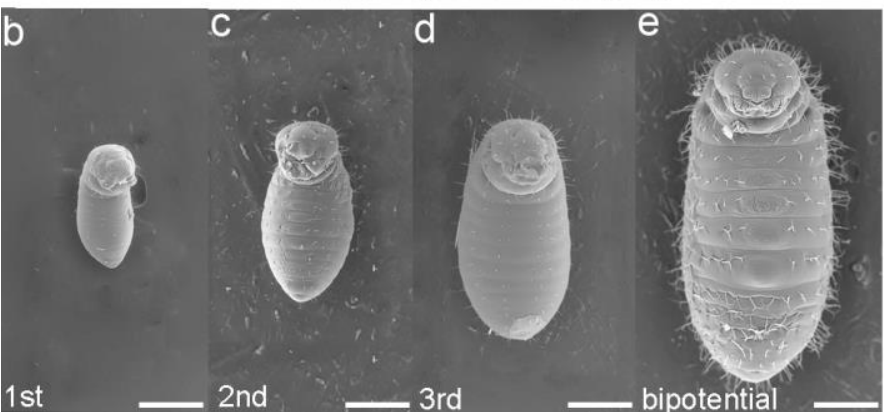

f $g$

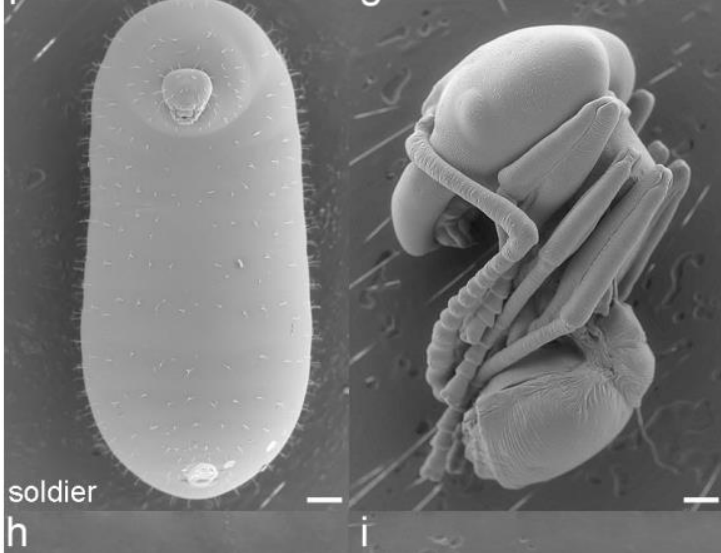

h

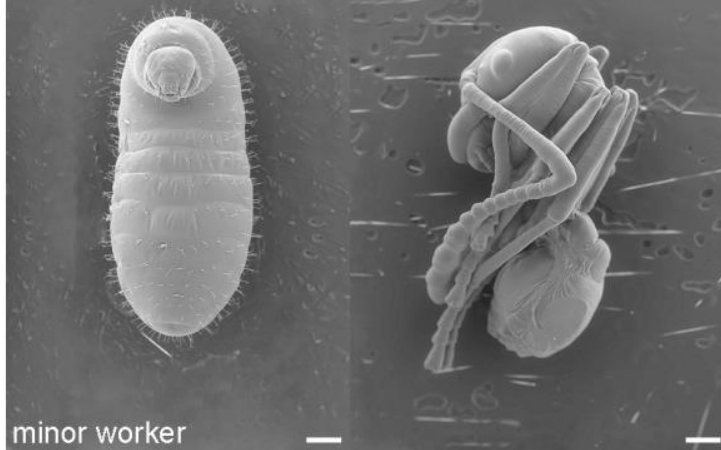

Figure 2. Larval development in P. hyatti generates discrete worker subcastes. a) Larval

542 capsule width $(\mu \mathrm{m})$ versus larval length $(\mu \mathrm{m})$ identifies four larval instars and differentiation of

543 worker subcastes $(n=154) . b-e, f, h)$ Representative individuals of the first $(b)$, second $(c)$, third

544 (d), and fourth $(e, f, h)$ instars in ventral view. The fourth instar includes bipotential larvae (e),

545 minor worker (h), and soldier (f) larvae. Minor worker and soldier larvae represent terminal

546 larvae immediately before metamorphosis. g, i: Representative pupal samples of a soldier (g)

547 and minor worker (i) in left lateral view. Scale bars indicate $200 \mu \mathrm{m}$. 
a

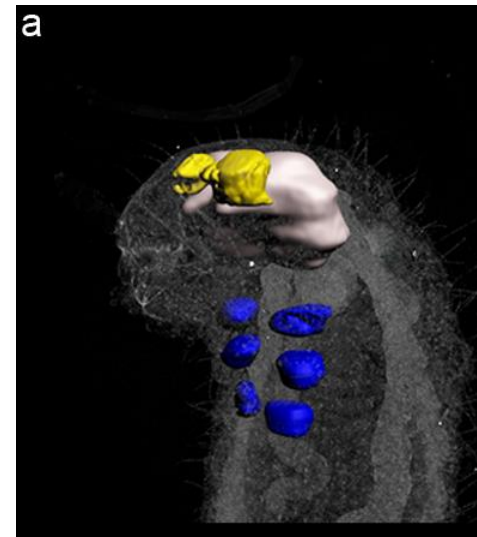

a'

$1033.43 \mu \mathrm{m}$

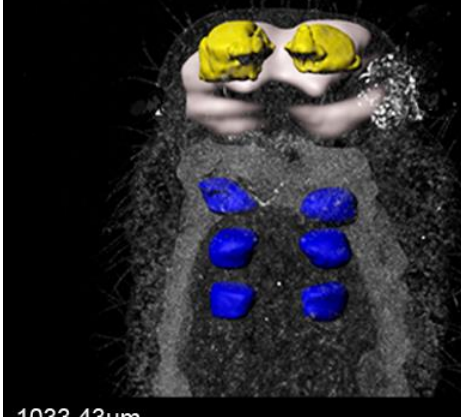

b

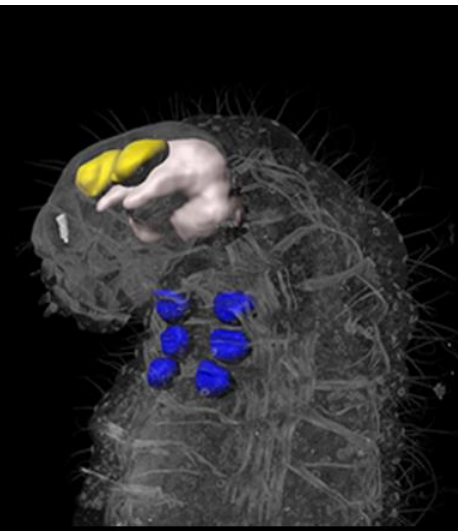

b'

Figure 3. 3D nano-CT analysis of imaginal disc development in bipotential larvae. Left lateral

$553(a-c)$ and ventral $\left(a^{\prime}-c^{\prime}\right)$ views of 3D nano-CT reconstructions of bipotential larvae: $1033.43 \mu \mathrm{m}$

$554\left(a, a^{\prime}\right), 1249.69\left(b, b^{\prime}\right)$, and $1527.61 \mu m\left(c, c^{\prime}\right)$ in length. Blue represents leg imaginal discs, yellow

555 represents eye-antennal discs, and white represents the larval brain. Larval length in $\mu \mathrm{m}$ is

556 indicated in the bottom left corner. Rudimentary forewing discs are undetectable. Images to

557 scale, scale bar $=70 \mu \mathrm{m}$. 

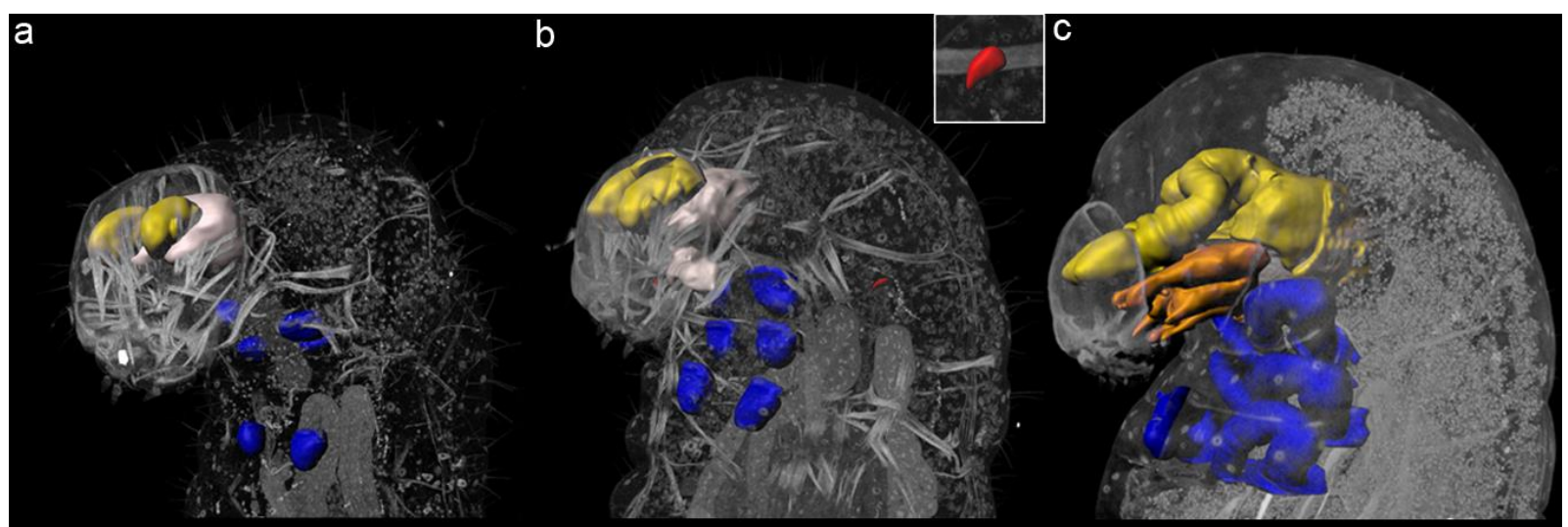

a'

b'

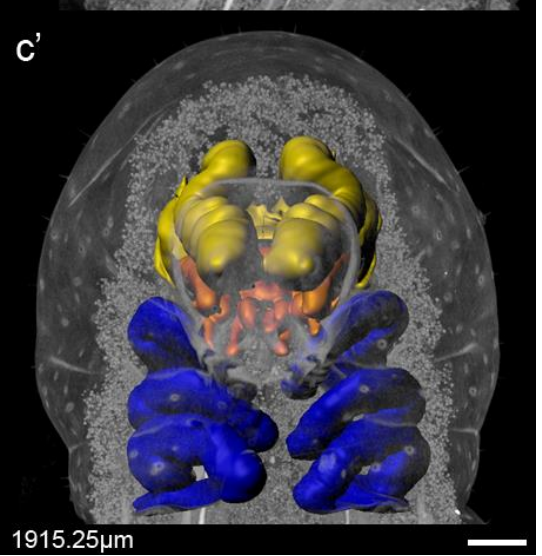

Figure 4. 3D nano-CT analysis of imaginal disc development in minor worker larvae. Left lateral $(\mathrm{a}-\mathrm{c})$ and ventral $\left(\mathrm{a}^{\prime}-\mathrm{c}^{\prime}\right)$ views of 3D nano-CT reconstructions of minor worker-destined $\left(a, a^{\prime}, b, b^{\prime}\right)$ and terminal minor worker (MW; c, c') larvae: 1634.56 $4 \mathrm{~m}\left(\mathrm{a}, \mathrm{a}^{\prime}\right), 1723.86 \mu \mathrm{m}\left(\mathrm{b}, \mathrm{b}^{\prime}\right)$, and $1915.25 \mu \mathrm{m}\left(\mathrm{c}, \mathrm{c}^{\prime}\right)$ in length. Inset in (b) is magnified to show rudimentary forewing discs in sample (b, b'). Larval length in $\mu \mathrm{m}$ is indicated in the bottom left corner. Blue represents leg imaginal discs, yellow represents eye-antennal discs, orange in (c, $c^{\prime}$ ) represents labial and/or clypeo-labral discs, and white represents the larval brain. Note that in $\left(b, b^{\prime}\right)$ the distinct mass

571 labeled white under the main larval brain (also white) may correspond to the labial and/or

572 clypeo-labral disc before its differentiation into clear mouthparts. However, the resolution of

573 our scan was not sufficient to confirm whether this distinct mass labeled white under the main

574 larval brain represents undifferentiated mouthparts and therefore should be labeled orange.

575 Rudimentary forewing discs were undetectable in $\left(a, a^{\prime}\right)$ and $\left(c, c^{\prime}\right)$. Images comparisons to 576 scale, scale bar $=100 \mu \mathrm{m}$. 


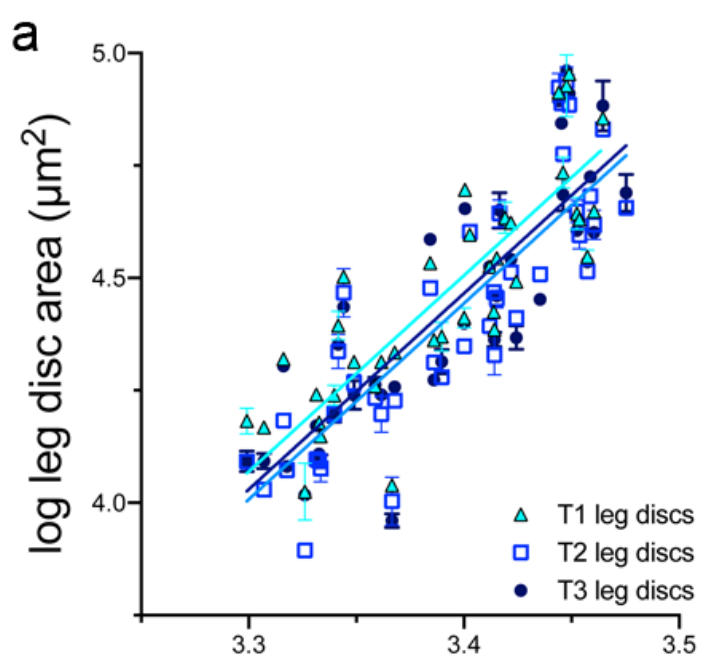

log larval length $(\mu \mathrm{m})$

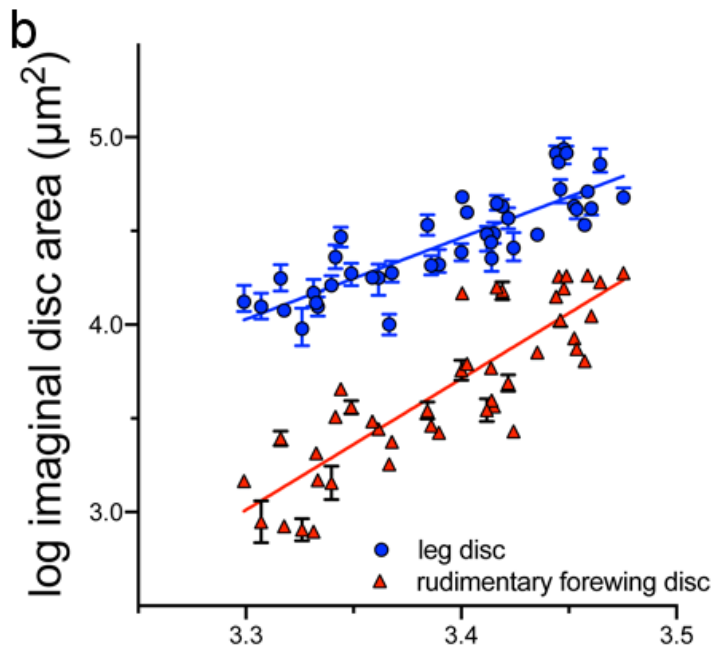

log larval length $(\mu \mathrm{m})$

581 Figure 5. Rudimentary forewing discs grow rapidly during soldier development. Imaginal disc

582 growth during soldier development: a) Linear regressions of log larval length $(\mu \mathrm{m})$ versus

583 average log leg disc area ( $\mu \mathrm{m} 2)$ separated based on thoracic segment identity (T1-T3) (n=43

584 larvae): T1 leg discs = turquoise circles $(n=37)$, T2 leg discs = blue squares $(n=41)$, T3 leg discs =

585 dark blue squares $(n=41)$ (slope: $F=0.2961, d f=2,113, P=0.7443$; $y$-intercept: $F=0.3184, d f=2$,

$586113, P=0.7280$ ). Best fit by a single curve $y=4.343 x-10.29$. b) Linear regressions of log larval

587 length $(\mu \mathrm{m})$ versus log area $(\mu \mathrm{m} 2)$ of soldier leg (blue) and rudimentary forewing (red) discs $(n=$ 58843 larvae) $(\mathrm{F}=11.51, \mathrm{df}=82, \mathrm{P}=0.011)$. Symbols indicate mean and error bars indicate range. 


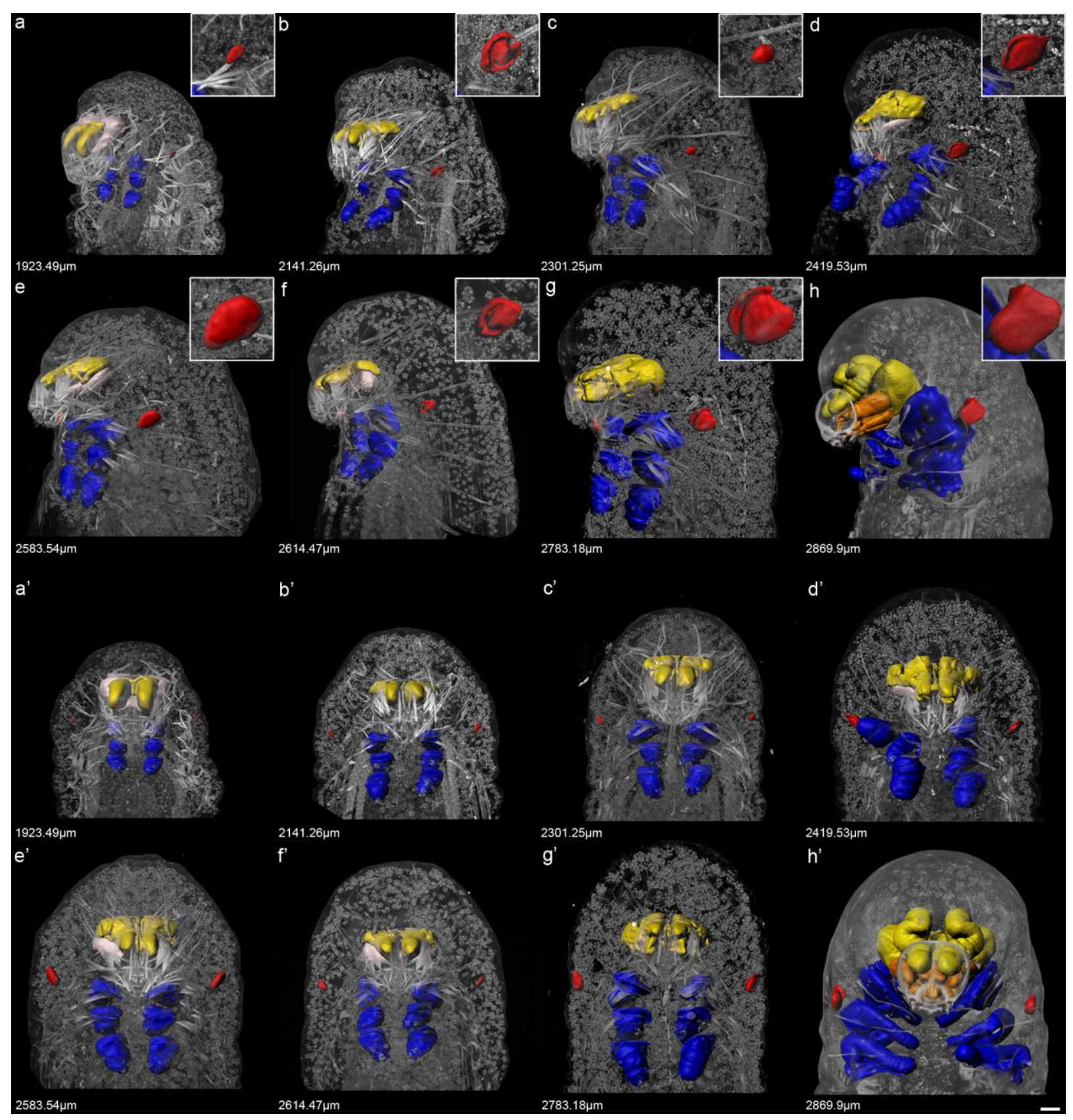

591 Figure 6. 3D nano-CT analysis of imaginal disc development in soldier larvae. Left lateral (a-h)

592 and ventral $\left(a^{\prime}-h^{\prime}\right)$ views of 3D nano-CT reconstructions of soldier-destined $\left(a-g, a^{\prime}-g^{\prime}\right)$ and

593 terminal soldier (SD; $\left.h, h^{\prime}\right)$ larvae: $1923.49 \mu \mathrm{m}\left(\mathrm{a}, \mathrm{a}^{\prime}\right), 2141.26 \mu \mathrm{m}\left(\mathrm{b}, \mathrm{b}^{\prime}\right), 2301.25 \mu \mathrm{m}\left(\mathrm{c}, \mathrm{c}^{\prime}\right)$,

$5942419.53 \mu \mathrm{m}\left(\mathrm{d}, \mathrm{d}^{\prime}\right), 2583.54 \mu \mathrm{m}\left(\mathrm{e}, \mathrm{e}^{\prime}\right), 2614.47 \mu \mathrm{m}\left(\mathrm{f}, \mathrm{f}^{\prime}\right), 2783.18 \mu \mathrm{m}\left(\mathrm{g}, \mathrm{g}^{\prime}\right)$, and $2869.9 \mu \mathrm{m}\left(\mathrm{h}, \mathrm{h}^{\prime}\right)$

595 in length. Insets in a-h show magnified rudimentary forewing discs in the corresponding

596 sample. Larval length in $\mu \mathrm{m}$ is indicated in the bottom left corner. Blue represents leg imaginal 
discs, yellow represents eye-antennal discs, orange in $\left(h, h^{\prime}\right)$ represents labial and/or clypeolabral discs, white represents the larval brain, and red represents rudimentary forewing discs.

599 Image comparisons to scale, scale bar $=100 \mu \mathrm{m}$.

600

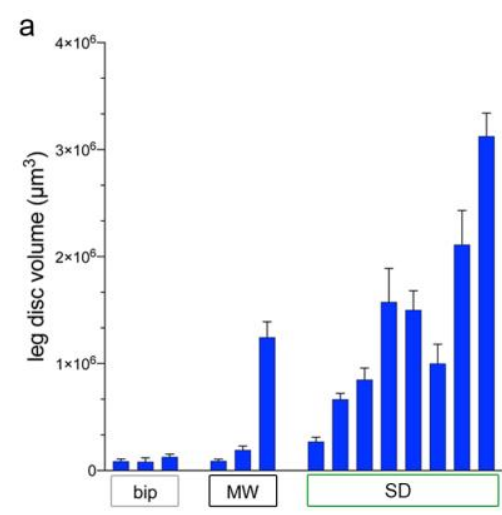

d

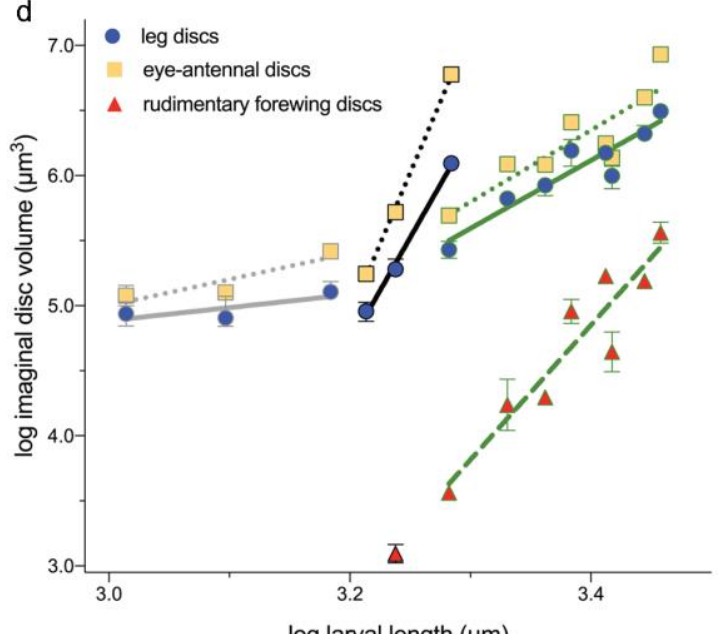

log larval length $(\mu \mathrm{m})$
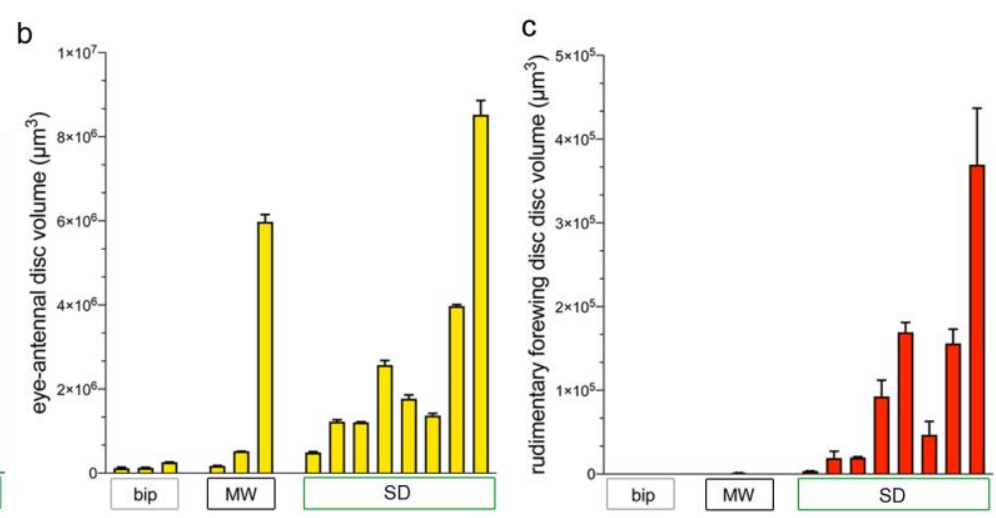

e

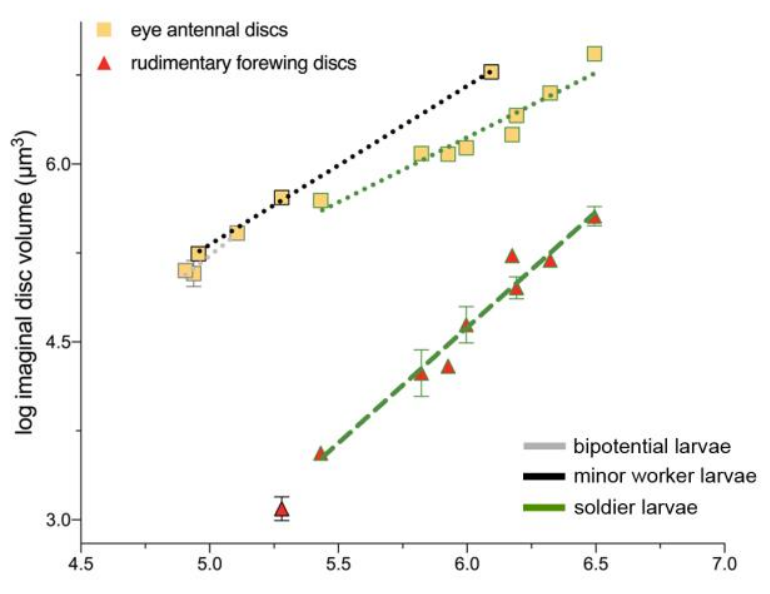

log leg disc volume $\left(\mu \mathrm{m}^{3}\right)$

Figure 7. Leg, eye-antennal, and rudimentary forewing disc growth across subcastes. a-c) Bar graphs showing imaginal disc volume $(\mu \mathrm{m} 3)$ versus larval length $(\mu \mathrm{m})$ grouped by developmental stage and subcaste. Bars show mean and error bars indicate range for: (a) leg disc volume $(\mu \mathrm{m} 3)$, (b) eye-antennal disc volume $(\mu \mathrm{m} 3)$, and (c) rudimentary forewing disc volume ( $\mu \mathrm{m} 3)$ grouped by bipotential (bip, grey), minor worker (MW, black), and soldier (SD, green) arranged by ascending larval length. d) Linear regressions of log larval length $(\mu \mathrm{m})$ versus log imaginal disc volume ( $\mu \mathrm{m} 3)$ grouped by subcaste. e) Linear regression of log leg disc volume $610(\mu \mathrm{m} 3)$ versus imaginal disc volume $(\mu \mathrm{m} 3)$ grouped by subcaste. Each point represents the mean 
611 and range within a larva. Blue circles represent leg discs (solid lines), yellow squares represent

612 eye-antennal discs (dotted lines), and red triangles represent rudimentary forewing discs

613 (dashed lines). Grey lines and outlines represent bipotential larvae, black lines and outlines

614 represent minor worker larvae, and green lines and outlines represent soldier larvae. The level

615 of significance was corrected for multiple comparisons using the Bonferroni correction.

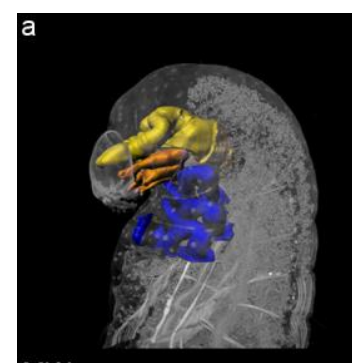

MW

c

c

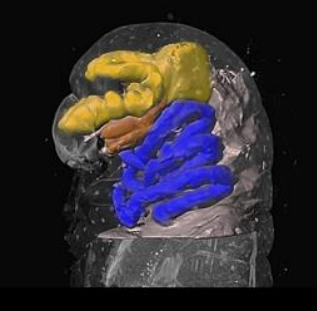

\section{a'}

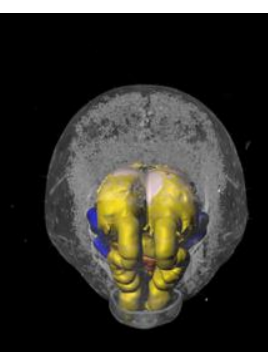

$\mathbf{C}^{\prime}$

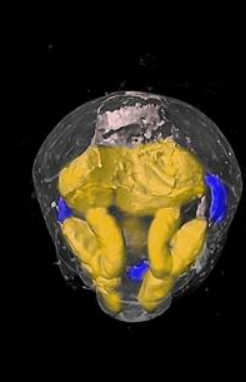

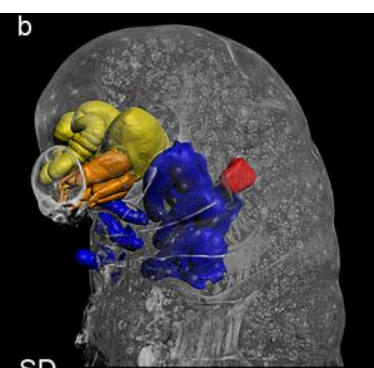

SD

d

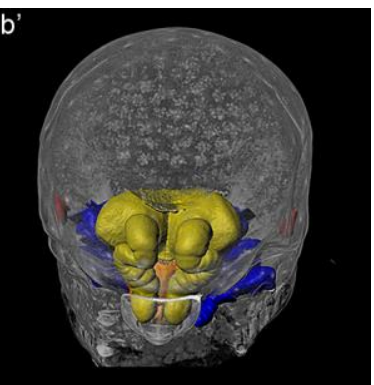

d'

Figure 8. Soldier eye-antennal discs grow larger and generate a wider head during

morphogenesis than in minor workers. Left lateral (a-d) and superior ( $\left.a^{\prime}-d^{\prime}\right)$ views of 3D nano-

621 CT reconstructions of terminal minor worker (MW) $\left(a, a^{\prime}\right)$, terminal soldier (SD) larvae $\left(b, b^{\prime}\right)$,

622 minor worker prepupae $\left(c, c^{\prime}\right)$, and soldier prepupae $\left(d, d^{\prime}\right)$. Blue represents leg imaginal discs,

623 yellow represents eye-antennal discs, orange represents labial and/or clypeolabral discs, white

624 represents the larval brain in $\left(a-b^{\prime}\right)$, and red represents rudimentary forewing discs. Images to

625 scale, scale bar $=100 \mu \mathrm{m}$.

626 


\section{References}

Abouheif, E., \& Wray, G. A. (2002). Evolution of the gene network underlying wing polyphenism in ants. Science, 297(5579), 249-252. doi:10.1126/science.1071468

Alvarado, S., Rajakumar, R., Abouheif, E., \& Szyf, M. (2015). Epigenetic variation in the Egfr gene generates quantitative variation in a complex trait in ants. Nature Communications, 6 , 6513. doi:10.1038/ncomms7513

Andersen, D. S., Colombani, J., \& Leopold, P. (2013). Coordination of organ growth: principles and outstanding questions from the world of insects. Trends in Cell Biology, 23(7), 336344. doi:10.1016/j.tcb.2013.03.005

Bhatkar, A., \& Whitcomb, W. H. (1970). Artificial diet for rearing various species of ants. The Florida Entomologist, 53(4), 229-232.

Bowsher, J. H., Wray, G. A., \& Abouheif, E. (2007). Growth and patterning are evolutionarily dissociated in the vestigial wing discs of workers of the red imported fire ant, Solenopsis invicta. J Exp Zool B Mol Dev Evol, 308(6), 769-776. doi:10.1002/jez.b.21200

Brian, M. V. (1963). Studies of caste differentiation in Myrmica rubra L. 6. Factors influencing the course of female development in the early third instar. Insectes Sociaux, 10, 91-102.

Busse, S. M., McMillen, P. T., \& Levin, M. (2018). Cross-limb communication during Xenopus hindlimb regenerative response: non-local bioelectric injury signals. Development, 145(19). doi:10.1242/dev.164210

Corona, M., Libbrecht, R., \& Wheeler, D. E. (2016). Molecular mechanisms of phenotypic plasticity in social insects. Current Opinion in Insect Science, 13, 55-60. doi:10.1016/j.cois.2015.12.003

Dewitz, H. (1878). Beitrage zur postembryonalen Gliedmassenbildung bei den Insekten. Z. Wiss. Zool. , 30, 78- 105.

Dominguez, M., \& Casares, F. (2005). Organ specification-growth control connection: new insights from the Drosophila eye-antennal disc. Developmental dynamics, 232(3), 673684. doi:10.1002/dvdy.20311

Dong, P. D., Dicks, J. S., \& Panganiban, G. (2002). Distal-less and homothorax regulate multiple targets to pattern the Drosophila antenna. Development, 129(8), 1967-1974.

Droujinine, I. A., \& Perrimon, N. (2016). Interorgan communication pathways in physiology: Focus on Drosophila. Annual Review of Genetics, 50, 539-570. doi:10.1146/annurevgenet-121415-122024 
671

672

673

674

675

676

677

678

679

680

681

682

683

684

685

686

687

688

689

690

691

692

693

694

695

696

697

698

699

700

701

702

703

704

705

706

707

708

709

710

711

712

713

714

Economo, E. P., Klimov, P., Sarnat, E. M., Guenard, B., Weiser, M. D., Lecroq, B., \& Knowles, L. L. (2015). Global phylogenetic structure of the hyperdiverse ant genus Pheidole reveals the repeated evolution of macroecological patterns. Proc Biol Sci, 282(1798), 20141416. doi:10.1098/rspb.2014.1416

Emlen, D. J., \& Nijhout, H. F. (2000). The development and evolution of exaggerated morphologies in insects. Annual Review of Entomology, 45, 661-708.

Emlen, D. J., Warren, I. A., Johns, A., Dworkin, I., \& Lavine, L. C. (2012). A mechanism of extreme growth and reliable signaling in sexually selected ornaments and weapens. Science, 337, 860-864.

Engel, M. S. (2015). Insect evolution. Curr Biol, 25(19), R868-872. doi:10.1016/j.cub.2015.07.059

Fjerdingstad, E. J., \& Crozier, R. H. (2006). The evolution of worker caste diversity in social insects. Am Nat, 167(3), 390-400. doi:10.1086/499545

Freener, D. H. J. (1987). Response of Pheidole morrisi to two species of enemy ants, and a general model of defense behavior in Pheidole (Hymenoptera: Formicidae). Journal of Kansas Entomological Society, 60(4), 569-575.

Fristrom, D. (1993). The metamorphic development of the adult epidermis. The Development of Drosophila melanogaster, 2, 843-897.

Garcia-Bellido, A., \& Merriam, J. R. (1969). Cell lineage of the imaginal discs in Drosophila Gynandromorphs. J. Exp. Zool., 170, 61-76.

Gontijo, A. M., \& Garelli, A. (2018). The biology and evolution of the Dilp8-Lgr3 pathway: A relaxin-like pathway coupling tissue growth and developmental timing control. Mech Dev, 154, 44-50. doi:10.1016/j.mod.2018.04.005

Hall, M. J., Simonsen, T. J., \& Martin-Vega, D. (2017). The 'dance' of life: visualizing metamorphosis during pupation in the blow fly Calliphora vicina by X-ray video imaging and micro-computed tomography. R Soc Open Sci, 4(1), 160699.

doi:10.1098/rsos.160699

Hariharan, I. K. (2012). How growth abnormalities delay "puberty" in Drosophila. Sci Signal, 5(229), pe27. doi:10.1126/scisignal.2003238

Haynie, J. L., \& Bryant, P. J. (1986). Development of the eye-antenna imaginal disk and morphogenesis of the adult head in Drosophila-Melanogaster. Journal of Experimental Zoology, 237(3), 293-308. 
Held, L. I. J. (2002). Imaginal Discs: The Genetic and Cellular Logic of Pattern Formation. Cambridge: Cambridge University Press.

Hölldobler, B., \& Wilson, E. O. (2009). The Superorganism. New York: W.W Norton \& Company Inc.

Holley, J. A. C., Moreau, C. S., Laird, J. G., \& Suarez, A. V. (2016). Subcaste-specific evolution of head size in the ant genus Pheidole. Biological Journal of the Linnean Society, 118(3), 472-485. doi:10.1111/bij.12769

Keller, R. A., Peeters, C., \& Beldade, P. (2014). Evolution of thorax architecture in ant castes highlights trade-off between flight and ground behaviors. Elife, 3, e01539. doi:10.7554/eLife.01539

Klingenberg, C. P., \& Nijhout, H. F. (1998). Competition among growing organs and developmental control of morphological asymmetry. Proceedings of the Royal Society BBiological Sciences, 265(1401), 1135-1139. doi:DOI 10.1098/rspb.1998.0409

Koch, Sophie, and Ehab Abouheif. "The Coordination of Insect Imaginal Discs and the Regulation and Evolution of Complex Worker Caste Systems of Ants." 197-224, 2019.

Kumar, J. P. (2018). The fly eye: Through the looking glass. Developmental dynamics, 247(1), 111-123. doi:10.1002/dvdy.24585

Kumar, K., Ouweneel, W. J., \& Faber, J. (1979). Differentiation capacities of the labial imaginal disc of Drosophila melanogaster. Wilhelm Roux's Archives of Developmental Biology, 186(1), 51-64. doi:10.1007/BF00848107

Lillico-Ouachour, A., \& Abouheif, E. (2017). Regulation, development, and evolution of caste ratios in the hyperdiverse ant genus Pheidole. Curr Opin Insect Sci, 19, 43-51. doi:10.1016/j.cois.2016.11.003

Londe, S., T. Monnin, R. Cornette, V. Debat, B. L. Fisher, and M. Molet. "Phenotypic Plasticity and Modularity Allow for the Production of Novel Mosaic Phenotypes in Ants." Evodevo 6 (2015): 36. https://doi.org/10.1186/s13227-015-0031-5.

Madhavan, K., \& Schneiderman, H. A. (1969). Hormonal Control of Imaginal Disc Regeneration in Galleria Mellonella (Lepidoptera). Biological Bulletin, 137(2), 321-331. doi:Doi $10.2307 / 1540104$

Mertl, A. L., \& Traniello, J. F. A. (2009). Behavioral evolution in the major worker subcaste of twig-nesting Pheidole (Hymenoptera: Formicidae): does morphological specialization influence task plasticity? Behavioral Ecology and Sociobiology, 63(10), 1411-1426. doi:10.1007/s00265-009-0797-3 
Metscher, B. D. (2009a). MicroCT for comparative morphology: simple staining methods allow high-contrast 3D imaging of diverse non-mineralized animal tissues. BMC Physiol, 9, 11. doi:10.1186/1472-6793-9-11

Metscher, B. D. (2009b). MicroCT for developmental biology: a versatile tool for high-contrast 3D imaging at histological resolutions. Dev Dyn, 238(3), 632-640. doi:10.1002/dvdy.21857

Metzl, C., Wheeler, D. E., \& Abouheif, E. (2018). Wilhelm Goetsch (1887 - 1960): pioneering studies on the development and evolution of the soldier caste in social insects. Myrmecological News, 26.

Milner, M. J., \& Haynie, J. L. (1979). Fusion of Drosophila eye-antennal imaginal discs during differentiation in Vitro. Wilhelm Roux Archiv, 185, 363-370.

Miyazaki, S., Murakami, T., Kubo, T., Azuma, N., Higashi, S., \& Miura, T. (2010). Ergatoid queen development in the ant Myrmecina nipponica: modular and heterochronic regulation of caste differentiation. Proc Biol Sci, 277(1690), 1953-1961. doi:10.1098/rspb.2010.0142

Molet, M., D. E. Wheeler, and C. Peeters. "Evolution of Novel Mosaic Castes in Ants: Modularity, Phenotypic Plasticity, and Colonial Buffering." Am Nat 180, no. 3 (Sep 2012): 328-41. https://doi.org/10.1086/667368.

Nijhout, H. F., \& Emlen, D. J. (1998). Competition among body parts in the development and evolution of insect morphology. Proceedings of the National Academy of Sciences of the United States of America, 95(7), 3685-3689. doi:DOI 10.1073/pnas.95.7.3685

Panganiban, G., Sebring, A., Nagy, L., \& Carroll, S. (1995). The development of crustacean limbs and the evolution of arthropods. Science, 270, 1363-1366.

Parker, N. F., \& Shingleton, A. W. (2011). The coordination of growth among Drosophila organs in response to localized growth-perturbation. Dev Biol, 357(2), 318-325. doi:10.1016/j.ydbio.2011.07.002

Passera, L. (1974). Différenciation des soldats chez la Fourmi Pheidole pallidula Nyl. (Formicidae Myrmicinae). Insectes Sociaux, 21(1), 71-86.

Passera, L., \& Suzzoni, J. P. (1978). Obtaining of sexual brood after JH treatment in ant Pheidole-pallidula -(NYL) (Hymenoptera, Formicidae). Comptes Rendus Hebdomadaires Des Seances De L Academie Des Sciences Serie D, 286(8), 615-618.

Patel, N. H. (1994). Imaging neuronal subsets and other cell types in whole-mount Drosophila embryos and larvae using antibody probes. Methods Cell Biol., 44, 445-487. 
Penick, C. A., \& Liebig, J. (2012). Regulation of queen development through worker aggression in a predatory ant. Behavioral Ecology, 23(5), 992-998. doi:10.1093/beheco/ars062

Pfeiffer, M., Nais, J., \& Linsenmair, K. E. (2006). Worker size and seed size selection in 'seed'collecting ant ensembles (Hymenoptera: Formicidae) in primary rain forests on Borneo. Journal of Tropical Ecology, 22(06), 685-693. doi:10.1017/s0266467406003622

Pie, M. R., \& Traniello, J. F. A. (2007). Morphological evolution in a hyperdiverse clade: the ant genus Pheidole. Journal of Zoology, 271(1), 99-109. doi:10.1111/j.14697998.2006.00239.x

Powell, S., \& Clark, E. (2004). Combat between large derived societies: A subterranean army ant established as a predator of mature leaf-cutting ant colonies. Insectes Sociaux, 51(4), 342-351. doi:10.1007/s00040-004-0752-2

Rajakumar, R., Koch, S., Couture, M., Fave, M.-J., Lilico-Ouachour, A., Chen, T.,Abouheif, E. (2018). Social regulation of a rudimentary organ generates complex worker caste systems in ants. Nature, 562, 574-577.

Rajakumar, R., Mauro, D. S., Dijkstra, M. B., Huang, M. H., Wheeler, D. E., Hiou-Tim, F., Abouheif, E. (2012). Ancestral developmental potential facilitates parallel evolution in ants. Science, 335(6064), 79-82. doi:10.1126/science.1211451

Rosello-Diez, A., Madisen, L., Bastide, S., Zeng, H., \& Joyner, A. L. (2018). Cell-nonautonomous local and systemic responses to cell arrest enable long-bone catch-up growth in developing mice. PLoS Biol, 16(6), e2005086. doi:10.1371/journal.pbio.2005086

Sameshima, S., Miura, T., \& Matsumoto, T. (2004a). Interspecific variation in the pupal-wing buds of major workers in the ant genus Pheidole (Hymenoptera: Formicidae). Sociobiology, 44(1), 101-107.

Sameshima, S. Y., Miura, T., \& Matsumoto, T. (2004b). Wing disc development during caste differentiation in the ant Pheidole megacephala (Hymenoptera: Formicidae). Evolution \& Development, 6(5), 336-341. doi:10.1111/j.1525-142X.2004.04041.

Schoborg, T., Smith, S., Smith, L., Morris, H. D., \& Rusan, N. M. (2018). Micro computed tomography as an accessible imaging platform for exploring organism development and human disease modeling. bioRxiv. doi:10.1101/391300

Schoeller, J. (1964). Recherches descriptives et experimentales sur la cephalogenese de Calliphora erythrocephala (Meigen) au cours des developpements embryonnaire et postembryonnaire. In L. Fage, G. Petit, \& G. Teissier (Eds.), Archives de Zoologie 
Experimentales et Generale (Vol. 103, pp. 1-216). Paris: Centre National de la Recherche Scientifique.

Shbailat, S. J., \& Abouheif, E. (2013). The wing-patterning network in the wingless castes of Myrmicine and Formicine ant species is a mix of evolutionarily labile and non-labile genes. J Exp Zool B Mol Dev Evol, 320(2), 74-83. doi:10.1002/jez.b.22482

Shbailat, S. J., Khila, A., \& Abouheif, E. (2010). Correlations between spatiotemporal changes in gene expression and apoptosis underlie wing polyphenism in the ant Pheidole morrisi. Evol. Dev., 12(6), 580-591. doi:DOI 10.1111/j.1525-142X.2010.00443.x

Shingleton, A. W., \& Frankino, W. A. (2018). The (ongoing) problem of relative growth. Current Opinion in Insect Science, 25, 9-19. doi:10.1016/j.cois.2017.10.001

Solis, D. R., Fox, E. G., Kato, L. M., de Jesus, C. M., Yabuki, A. T., Campos, A. E., \& Bueno, O. C. (2010). Morphological description of the immatures of the ant, Monomorium floricola. J Insect Sci, 10, 15. doi:10.1673/031.010.1501

Trible, W., \& Kronauer, D. J. (2017). Caste development and evolution in ants: it's all about size. J Exp Biol, 220(Pt 1), 53-62. doi:10.1242/jeb.145292

Trible, W., \& Kronauer, D. J. C. (2021). Hourglass Model for Developmental Evolution of Ant Castes. Trends in Ecology \& Evolution, 36(2), 100-103. doi:10.1016/j.tree.2020.11.010

Truman, J. W., \& Riddiford, L. M. (1999). The origins of insect metamorphosis. Nature, 401, 447452.

Vallejo, D. M., Juarez-Carreno, S., Bolivar, J., Morante, J., \& Dominguez, M. (2015). A brain circuit that synchronizes growth and maturation revealed through Dilp8 binding to Lgr3. Science, 350(6262), aac6767. doi:10.1126/10.1126/science.aac6767

Watanabe, H., Yanase, Y., \& Fujii, Y. (2017). Nondestructive evaluation of egg-to-adult development and feeding behavior of the bamboo powderpost beetle Dinoderus minutus using X-ray computed tomography. Journal of Wood Science, 63(5), 506-513. doi:10.1007/s10086-017-1642-3

West-Eberhard, M. J. (2003). Developmental plasticity and evolution. Oxford; New York: Oxford University Press.

Wheeler, D. E. (1986). Developmental and physiological determinants of caste in social hymenoptera - evolutionary implications. Am. Nat., 128(1), 13-34.

Wheeler, D. E., \& Nijhout, H. F. (1981a). Imaginal wing discs in larvae of the soldier caste of Pheidole bicarinata vinelandica forel (Hymenoptera: Formicidae). International Journal of Insect Morphology and Embryology, 10(2), 131-139. 
890

891

892

893

894

895

896

897

898

899

900

901

902

903

904

905

906

907

908

909

910

911

912

913

914

915

916

917

918

919

920

921

922

923

924

Wheeler, D. E., \& Nijhout, H. F. (1981b). Soldier determination in ants: new role for juvenilehormone. Science, 213(4505), 361-363.

Wheeler, D. E., \& Nijhout, H. F. (1983). Soldier determination in Pheidole bicarinata: Effect of methoprene on caste and size within castes. Journal of Insect Physiology, 29(11), 847854.

Wheeler, D. E., \& Nijhout, H. F. (1984). Soldier determination in Pheidole bicarinata: Inhibition by adult soldiers. Journal of Insect Physiology, 30(2), 127-135.

Wheeler, G. C., \& Wheeler, J. (1976). Ant larvae: review and synthesis. Entomol. Soc. Washington., 7, 1-108.

Williams, J. A., \& Carroll, S. (1993). The origing, patterning and evolution of insect appendages. Bioessays, 15(9).

Wills, B. D., Powell, S., Rivera, M. D., \& Suarez, A. V. (2018). Correlates and consequences of worker polymorphism in ants. Annual Review of Entomology, 63, 575-598. doi:10.1146/annurev-ento-020117-043357

Wilson, E. O. (1953). The origin and evolution of polymorphism in ants. Quarterly Review of Biology, 28(2), 136-156.

Wilson, E. O. (1954). A new interpretation of the frequency curves associated with ant polymorphism. Insectes Sociaux, 1(1), 75-80.

Wilson, E. O. (1984). The relation between caste ratios and division of labor in the ant genus Pheidole (Hymenoptera: Formicidae). Behavioral Ecology and Sociobiology, 16(1), 89-98. doi:10.1007/BF00293108

Wilson, E. O. (2003). Pheidole in the New World: Harvard University Press.

Yang, A. S. (2001). Modularity, evolvability, and adaptive radiations: a comparison of the hemiand holometabolous insects. Evolution \& Development, 3(2), 59-72. 